The energy of gamma rays emitted after neutron capture can now be measured with parts-per-million precision. The quest for this precision, which allows us to measure tiny Doppler effects caused by recoil after the emission of gamma rays or neutrinos, has given rise to new applications in nuclear and solid state physics

Jan Jolie, Switzerland

\title{
Gamma Ray Spectroscopy with Ultra-High Precision
}

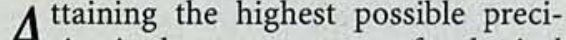
$A_{\text {sion in the measurement of a physical }}$ quantity has always been one of the major goals of experimental physicists. Though this quest for precision is often a frustrating and not necessarily an immediately rewarding task (no technological applications are generally envisaged) physics has always progressed by improvements in precision. The quest for precision leads to the development of better instruments and of new experimental methods. Improved precision might also reveal unexpected effects, the kind that often stir new theoretical and experimental developments.
\end{abstract}

\section{Gamma ray standards}

In the case of gamma rays, which are photons with energies above $100 \mathrm{keV}$ or wavelengths below one tenth of an angström, the task of measuring the energy precisely is especially difficult. Due to their high penetration power these very high energy photons do not deposit their energy easily in a detector. The common detectors today are relatively large volume solid state detectors. These $\mathrm{Ge}$ and $\mathrm{Si}$ detectors measure the gamma ray energy with a reasonable efficiency, but cannot attain an energy resolution better than
$0.1 \%$. This will not help us to measure "standards".

A more precise way of determining the energy consists of measuring the wavelength using crystal diffraction. But again, there are many difficulties typical of gamma rays which need to be surmounted. Low crystal reflectivity forces the use of very strong gamma ray sources. Because there are no possibilities for making reflecting planes which are spaced closer than a few angströms, the application of $n \lambda=2 d \sin \left(\theta_{B}\right)$, the Bragg law, shows that for first order diffraction of a $1 \mathrm{MeV}$ gamma ray the Bragg angle $\theta_{B}$ is below a quarter of a degree. You thus need to measure very small angles with parts-per-million (ppm) precision. At the same time, you need to know the only other experimental quantity appearing in the Bragg law, the lattice spacing $d$, with an even higher precision.

During the early seventies R.D. Deslattes, E.G. Kessler and coworkers at the National Bureau of Standards (now the National Institute for Science and Technology, NIST, Washington) attacked the problem of a gamma ray standard. In order to increase the accuracy of the energy measurement they adopted a three-step approach. In the first step the visible light standard was used to measure the silicon repeat distance $d$ in a "master" crystal. This was performed using a ${ }^{129} \mathrm{I}_{2}$ stabilized ${ }^{3} \mathrm{He}^{22} \mathrm{Ne}$ laser which was linked via the Rydberg constant to the then valid ${ }^{86} \mathrm{Kr}$ standard definition of the metre. Using Fabry-Perot interferometry the lattice spacing $d$ between the two [220] crystal planes of hyperpure silicon could be determined with an error of a few hundredths of a femtometre. (An even more accurate value has been obtained at the PTB, the German institute of standards in Braunschweig, by P. Becker and coworkers). Once the lattice spacing of the "master" crystal had been determined to 0.1 ppm accuracy, the second step concerned the use of this distance to calibrate other crystals. These calibrated crystals were then mounted on the two-axis flat crystal spectrometer GAMS 4 . This instrument is described in the box.

By measuring the absolute angle $2 \theta_{B}$ no other reference is needed other than the lattice spacing, and the gamma ray wavelength is thus obtained as a function of the definition of the metre. Accurate measurements sometimes have far reaching consequences for the determination of other fundamental constants. New standard lines measured in the 1970 (for the calibration of radioactive sources) allowed the solution of a discrepancy between QED and the measured x-ray transitions of muonic atoms. This discrepancy was not caused by experimental errors but by the inaccurate calibration lines used. Other important results were improved values for the mass of the negative pion and kaon.

After the installation of GAMS 4 at the high-flux reactor of the Institut Laue Langevin (ILL), under a NIST/ILL collaboration, gamma rays emitted after thermal neutron capture could be measured. Among these is the deuteron line for which the Bragg angle for first order diffraction on [400] Ge planes was determined to be $\theta_{B}=0.11294860(11)^{\circ}$. Via the deuteron binding energy, this experiment yielded a very accurate determination of the neutron mass. (Recently the lattice
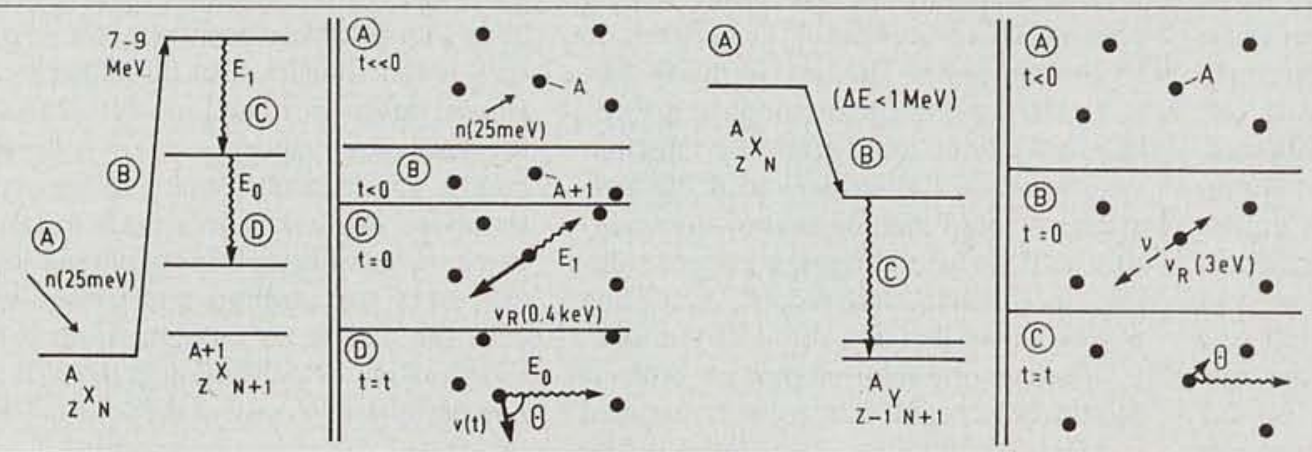

Fig 1 Comparison between the events which take place at the nuclear and atomic scale after thermal neutron capture (left) or electron capture (right) 


\section{The GAMS4 Spectrometer}

The two-axis flat crystal spectrometer GAMS4 consists of two highly perfect flat $\mathrm{Si}$ or Ge crystals of which the crystal planes are oriented such that gamma rays with a certain wavelength $\lambda$ can successively fulfil the Bragg-Laue condition (for diffraction of the gamma rays) for both crystals (in transmission). The high purity crystals are cut such that the flag available for diffraction is free of stress. Each crystal is mounted on a crystal table connected to one of the two rotation axes of the spectrometer. In order to measure the absolute angle a special Michelson interferometer is used, which can measure angles with a sensitivity of $10^{-10}$ radians. During the measurement, temperature and pressure are monitored and used to correct the optical path for wavelength shifts. As the angle sensitivity is so high, the only reference angle that can be measured is 360 degrees, which is done by measuring the angle in between the 24 faces of a fused silica optical polygon in fringes. After having been diffracted, the gamma rays pass a long collimator and enter a Ge detector. For each angle the peak corresponding to the gamma ray energy in the Ge spectrum is fitted. The total number of counts in this peak then gives the measured line profile as function of the diffraction angle with respect to the second crystal. Two-axis flat-crystal spectrometers have interesting geometrical properties that are essential to their use. Considering diffraction by the same order of reflection $\mathrm{n}$, the second crystal can fulfil the Bragg condition when being turned at $\theta_{B}$ or at $-\theta_{B}$ with respect to the incoming beam. From the angle $2 \theta_{B}$ the absolute wavelength of the gamma rays follows. This is the basis of the use of $\mathrm{GAMS}_{4}$ for the measurements of gamma ray standards. For the other applications of $\mathrm{GAMS}_{4}$, the introduction of the second crystal makes it possible to measure the energy dispersion of a gamma ray as well as the instrumental response at a certain angle. If the second crystal is placed parallel to the first one, then the angle under which all reflected gamma rays enter the second crystal is the same as the one under which they entered the first crystal (see figure). Consequently, gamma rays with slightly different energies, which are diffracted by the first crystal under different angles, will encounter the second crystal under the same angles again and can diffract again. On the contrary, if the crystal is not exactly parallel the Bragg-Laue condition is not fulfilled for any incident gamma ray. Thus by rocking the second crystal around $\theta_{B}$, one measures the exact instrumental response of both crystals. Therefore, the peak measured with parallel crystals is "non-dispersive". If one turns the second crystal to its mirror image with respect to the direction of the diffracted beam at $-\theta_{B}$ (see figure), then the gamma rays which had the exact energy $\theta_{B}$ for which the first crystal was oriented will find the second crystal placed exactly at the angle suited again for diffraction. However, all gamma rays with slightly different wavelengths $\lambda^{\prime}$ diffracted under a slightly-different angle $\theta_{B}$ ' by the first crystal find the second crystal at twice the angular difference $\theta_{B}-\theta_{B}$, As the conditions for diffraction are not fulfilled these gamma rays do not enter the detector. This geometry (of the crystals) is therefore called "dispersive". By rocking the second crystal, extra broadening, which is not related to instrumental effects, may be observed: Doppler broadening and natural line width. By measuring the angles $+\theta_{B}$ and $-\theta_{B}$ for which $n$th order reflection occurs on the second crystal one thus obtains both the wavelength $\lambda$ from the angle $2 \theta_{B}$ and the energy distribution by unfolding (thus subtracting) the instrumental response function measured at angle $+\theta_{B}$ from the distribution measured at $-\theta_{B}$. Because GAMS 4 only transmits gamma rays of energy $E_{0}$ which are incident on the crystals almost parallel to each other, the efficiency is of the order of $10^{-12}$ for low orders of reflection. Therefore since 1984 the spectrometer has been installed at the $58 \mathrm{MW}$ High-Flux Reactor of the Institute LaueLangevin in Grenoble, France. The combination of the highest neutron flux available today $\left( \pm 5.10^{14} n \mathrm{~cm}^{-2} \mathrm{~s}^{-1}\right)$ and an in-pile source changing facility placing the targets only $50 \mathrm{~cm}$ from the reactor core allows getting sufficient gamma ray fluxes. spacing, deuteron line and neutron mass were remeasured yielding even more accurate values.)

\section{Doppler broadening}

In 1986 at the ILL, H.G. Börner realized that it would be possible to use GAMS 4 to measure tiny Doppler shifts in gamma ray energies. These are caused by the small recoil velocity, $v_{r}$, which an atomic nucleus with mass $M$ gets when it emits a primary gamma ray with energy $E_{\gamma}$ : $v_{r} / c=E_{\gamma} / M c^{2}$

These recoil velocities are of the order of $10^{-4}$ to $10^{-6}$ of the velocity of light, $c$, thus their Doppler effect on secondary transitions should be observable with GAMS4. Because there is no preferred direction for the emission of the primary gamma rays, the secondary gamma rays will show a Doppler broadening instead of a
Doppler shift. The first measurements performed using the ${ }^{48} \mathrm{Ti}(n, \gamma)$ reactionthe transformation of ${ }^{48} \mathrm{Ti}$ into ${ }^{49} \mathrm{Ti}$ by adding a neutron-revealed the expected effect, as well as a dependence of the broadening on the lifetime of the nucleus. With this first experiment and the analysis, which was also developed quickly by the ILL group, the Gamma Ray Induced Doppler broadening (GRID) technique was born.

To understand the basic principles of this technique consider the schematic representation shown in the left part of figure 1 . The capture of a thermal neutron having an energy of $25 \mathrm{meV}$ has no influence on the atomic scale, but leads on the nuclear scale to the formation of an highly excited isotope (typically about $8 \mathrm{MeV}$ ).

The newly created isotope will therefore quickly emit a primary gamma ray of several $\mathrm{MeV}$. At the moment of emission, which we define as $t=0$, on the atomic scale the atom starts to recoil in the opposite direction. The primary transition generally populates another excited state, which decays with a given lifetime $\tau$ by another gamma ray. Before the emission of this gamma ray the atom will have made collisions with neighbouring atoms and both the magnitude and direction of its velocity will have changed. At the moment of the second emission the projection of the atoms velocity onto the direction of emission is revealed by the measured Doppler shift. When observing many secondary gamma rays this will lead to a Doppler broadening that contains information about both the nuclear lifetime and the slowing down process (which occurs when the atom, having received a kick from a photon, then collides 


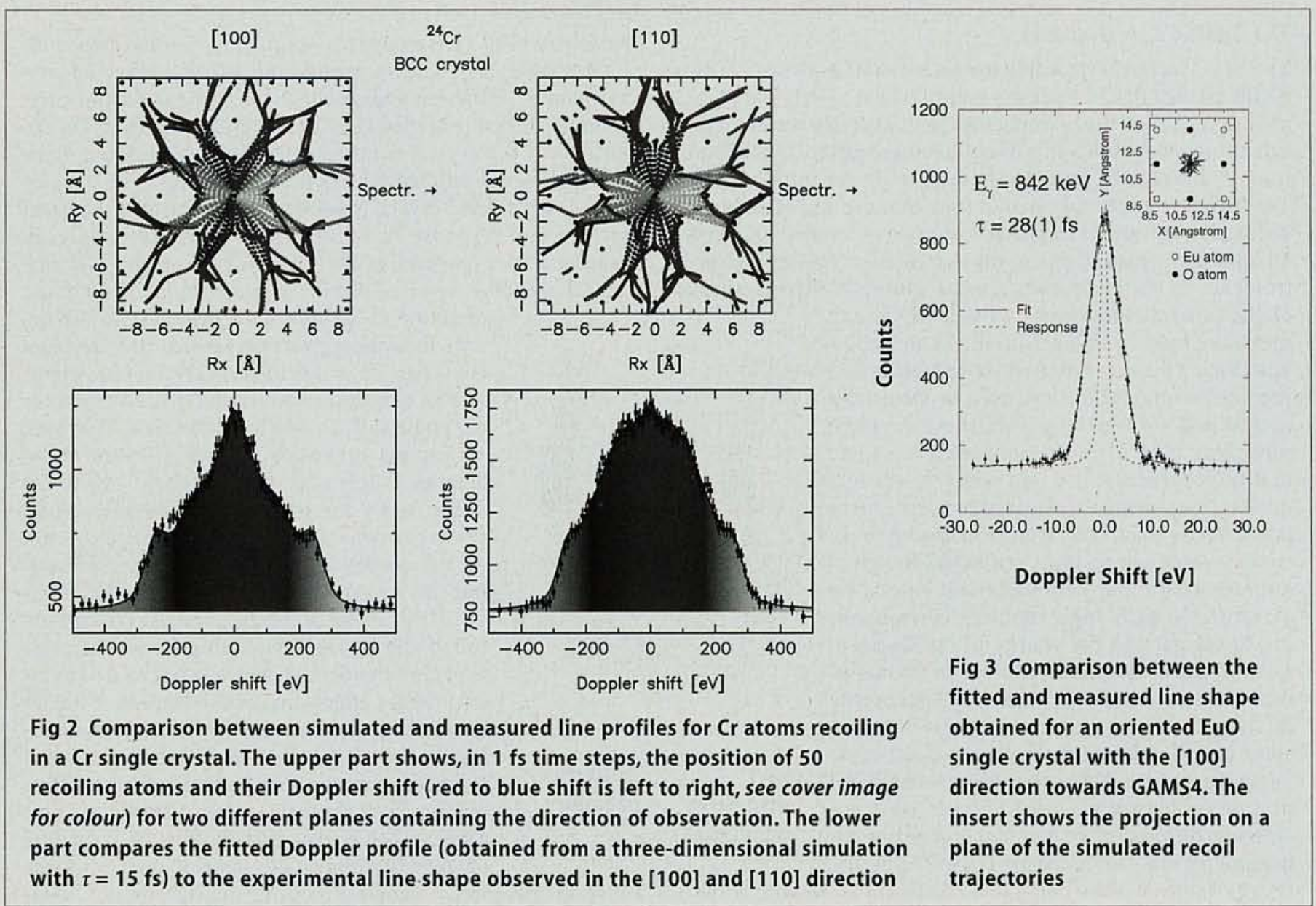

with other atoms). In a certain sense the radioactive decay law provides thus a sort of nuclear clock for the slowing-down process and vice versa. Besides gamma ray emission, other nuclear decay modes induce recoils. In the right part of figure 1, this is illustrated for electron capture. In electron capture the nucleus undergoes a beta decay of the form $p+e^{-} \rightarrow n+v$. On the atomic scale (to a first approximation) only a neutrino is emitted and thus the atom undergoes a well defined recoil in the opposite direction. Because the kinetic energy of the neutrino in pure electron capture has to be below $2 m_{e} c^{2}$, the Neutrino Induced Doppler (NID) broadening provides a way of obtaining small recoils of the order of a few eV in heavy nuclei. The small recoil does not permit a permanent displacement of the atom, but rather leads to oscillations of the atom about its equilibrium position. These can now be studied when the atom emits a gamma ray that will undergo a small Doppler shift. The high sensitivity of the GAMS 4 spectrometer quickly revealed that the thermal motion of the target atoms causes an additional Doppler broadening. This needs to be taken into account for a proper analysis of the data and has become one of the limiting factors of the GRID and NID methods.

\section{Nuclear structure results}

Initially, the main motivation for the elaboration of the GRID method concerned the measurement of short nuclear lifetimes. Because typical slowing down times are about 100 femtoseconds, lifetimes of femtoseconds ( $1 \mathrm{fs}=10^{-15} \mathrm{~s}$ ) to picoseconds ( $1 \mathrm{ps}=10^{-12} \mathrm{~s}$ ) can be extracted from the analysis of the measured line shape. No other technique is able to measure such short lifetimes after neutron capture. Therefore, the GRID method made it possible to measure lifetimes of low-spin states at relatively high excitation energies, as those states are predominantly reached via the $(n, \gamma)$ reaction. This new possibility allows for some hitherto unfeasible tests of nuclear models. In light nuclei, the application of the GRID method is rather straightforward because the primary transitions strongly "feed" the levels of interest-there are fewer levels to feed in light nuclei. Moreover, the recoils are "large" due to the small mass. Most of the data can be interpreted in this way using a mean free path approach (MFPA) which yields a simple and parameter free slowing down model even in complicated targets. Besides testing the shell model, the most important result obtained using the GRID method in light nuclei is the observation of mixed symmetry states in the $\mathrm{N}=42$ isotopes. This gave the first direct evidence that these excitations, formed by an out of phase motion of valence protons and neutrons, exist in vibrational nuclei. In heavy nuclei the application of the GRID method is more complicated due to the smaller recoil energies and the millions of excited states-we can not determine experimentally the population of a level. The "feeding" of the level under study needs to be simulated by statistical models of which the most commonly used for GRID is the one developed by F. Becvar of Charles University (Prague). The main consequence of the statistical feeding is an additional uncertainty on the lifetimes. Despite these serious disadvantages, GRID studies in medium-heavy and heavy nuclei have yielded several important results. This is because the theoretical understanding built up by low-energy nuclear spectroscopy can be tested by its predictions for higher-lying excitations. Those tests often yield answers on the microscopic foundations of the models. The heaviest nucleus investigated using the GRID technique is ${ }^{196} \mathrm{Pt}$. This 
atomic nucleus was considered to be the best example of a recent algebraic model for medium-heavy and heavy nuclei: the $\mathrm{O}(6)$ dynamical symmetry of the interacting boson model. However, this interpretation was attacked when it was shown that another symmetry corresponding to the vibrational $U(5)$ limit of the model could also reproduce its structure. To solve this dilemma the lifetime of the excited state $\mathrm{J}^{\pi}=0^{+}$at $1.4 \mathrm{MeV}$ needed to be determined, because its decay to lower states is forbidden in the $\mathrm{O}(6)$ limit and very collective in the $U(5)$ limit. The GRID measurement revealed that the lifetime of this state is longer than about 2 $\mathrm{ps}$, confirming the $\mathrm{O}(6)$ character of ${ }^{196} \mathrm{Pt}$. One of the major research fields for GRID in medium-heavy even-even nuclei is the investigation of multiphonon excitations. In the "collective model" these nuclei are treated as a quantum liquid which can undergo surface oscillations in the form of vibrations. The lowest modes available to the nucleus are the deformations called quadrupole and octupole vibrations. In spherical symmetric nuclei, one and two quadrupole phonon as well as octupole states have been known for many years. Using the GRID technique higher phonon excitations have been studied by measuring the absolute electromagnetic quadrupole transition rates connecting them to lower excitations. In ${ }^{114} \mathrm{Cd}$ three quadrupole phonon states and their interaction with two particle-four hole excitations (across the $\mathrm{z}=50$ closed shell) have been studied. In ${ }^{144} \mathrm{Nd}$ the GRID study of quadrupole-octupole coupled states revealed for the first time absolute transition rates which showed good agreement with 23-years old predictions. When the atomic nucleus is not spherical, but only axially symmetric, the harmonic quadrupole vibration splits into two basic excitations called the beta and gamma vibration. The first GRID study performed on deformed nuclei concerned the long-debated question of the existence of the double-gamma vibration. Microscopically, the collective vibrational modes have to be described in terms of the nuclear many-fermion problem. In the case of an axially deformed mean field, the available single-particle orbitals are only two-fold degenerate instead of $(2 j+1)$-fold. Due to the Pauli exclusion principle it was thus not clear whether a double gamma vibration also exists. The fingerprint of this excitation lies in the occurrence of collective decay to the single gamma vibration. The major experimental problem to settle this old question is that it involves the measurement of lifetimes of highly excited low-spin states. In ${ }^{168} \mathrm{Er}$ the first candi- date for a double gamma vibration is the eighteenth excited rotational band of which the lowest level is the eight excited $4^{+}$state situated at $2.055 \mathrm{MeV}$. This state is, however, well populated by thermal neutron capture and the GRID study was able to show that its decay and hence the double gamma vibration is collective. This experiment has spurred several new experiments that search for other double gamma vibrations.

\section{Molecular Dynamics}

For a more detailed analysis of the GRID data A. Kuronen and J. Keinonen (University of Helsinki) introduced Molecular Dynamics (MD) simulations of the slowing-down process. With these simulations it is possible to follow the recoiling atom during a few hundreds of femtoseconds by solving the Newtonian equations of motion in $0.5 \mathrm{fs}$ time steps. This is done in a simulation cell containing up to 1500 atoms. The line shape can then be constructed by simulating many recoils generated using Monte Carlo techniques. In general this method can be applied in the case of short lifetimes ( $\tau<100 \mathrm{fs}$ ) and light nuclei, where most states are predominantly directly fed. Recently, an important improvement of the method was obtained with the introduction of oriented single crystals as targets. This CrystalGRID method was developed by $M$. Jentschel (FZ-Rossendorf, ILL) based on an original idea by K.H. Heinig and D. Janssen. They argued that due to the regular stacking of atoms in a single crystal, the rate of slowing down in such targets depends on the direction of recoil, due to blocking and channeling by other atoms. By observing the emitted gamma rays from atoms recoiling in single crystals as a function of the crystal orientation different red and blue shifts of the gamma rays are observed. Therefore a fine structure appears on the line shape which can be simulated using the MD codes. First experiments on $\mathrm{Ti}$ recoiling in $\mathrm{SrTiO}_{3}$ revealed the expected effect. The observation of the fine structure yields a more precise determination of the slowingdown process and/or the lifetimes. With this observation GAMS 4 opens up once more new vistas for ultra-high resolution gamma ray spectroscopy.

\section{Interatomic potentials}

It was realized very early in the development of the GRID method that when the nuclear lifetime is known the method permits the study of the slowing down process. As the kinetic energies induced by the different emissions are very low ( 3 to $500 \mathrm{eV}$ ), these studies test the inter- atomic potentials in the bulk of solids in what was before an inaccessible energy region. This is possible because the GRID method provides both the extra energy and a signal able to traverse a lot of material. To study the interatomic potentials, the analysis has to be performed using MD techniques. One method consists of performing the simulations using different potentials. The fitted lifetimes then become control parameters indicating the goodness of the potentials when compared to the literature values. The main problem with this method resides in the last point, since the results obtained using GRID are often more precise than the ones from another nuclear method. Using Crystal-GRID, however, the amount of information in the line shape is maximized and it becomes possible to distinguish between the quality of the different descriptions of the line shapes. For instance, line shapes measured under different orientations should yield the same lifetime. Recently, our group and the ILL group were able to extract both the lifetime and the form of the interatomic potential by leastsquares fitting of the Crystal-GRID line profiles. The simulations and results of such experiments on $\mathrm{Cr}$ are shown in figure 2. With this possibility the study of the interatomic potentials becomes independent of the measurement of the lifetime with another technique. In some cases it is possible to investigate the interatomic potential at very low energies using Crystal-NID. The result of a recent study on oriented EuO single crystals, where the recoil energy is only $3 \mathrm{eV}$, is shown in figure 3 . One notices that the observed broadening still allows one to study the interactions between the atoms, via the simulation of the oscillations of the recoiling atom.

All these new techniques would not have been possible without the search for precision that motivated the development of the GAMS 4 spectrometer.

EN

The author is a first assistant at the Physics Institute of the University of Fribourg in Switzerland

Financial support by the Swiss National Science Fund and the Institute Laue-Langevin is acknowledged

\section{Further reading}

R.D. Deslattes, E.G. Kessler, W.C. Sauder, A. Henins Ann. of Phys. 129378 (1980) - H.G. Börner and J. Jolie, Topical review Journ. of Phys. G 19217 (1993) - A. Kuronen et al, Nucl. Phys. A 54959 (1992) - M. Jentschel et al, Nucl. Instr. and Meth B 115446 (1996) - N. Stritt et al, Phys. Rev. Lett. 782592 (1997) - Phys. Rev. B (March 1999) 